\title{
Assessment of education requirements for patients with rheumatoid arthritis, based on the Polish version of the Educational Needs Assessment Tool (Pol-ENAT), in the light of some health problems - A cross-sectional study
}

\author{
Matylda Sierakowska', Małgorzata Klepacka², Stanisław Sierakowski , \\ Katarzyna Pawlak-Buśs ${ }^{4}$, Piotr Leszczyński ${ }^{4}$, Maria Majdan ${ }^{5}$, Marzena Olesińska ${ }^{6}$, \\ Wojciech Romanowski ${ }^{7}$, Małgorzata Bykowska-Sochacka ${ }^{8}$, Sławomir Jeka9, \\ Justyna Sierakowska ${ }^{10}$, Mwidimi Ndosi ${ }^{11}$, Elżbieta Krajewska-Kułak ${ }^{1}$ \\ ${ }^{1}$ Department of Integrated Medical Care, Medical University, Bialystok, Poland \\ ${ }^{2}$ Department of Nephrology, Medical University, Bialystok, Poland \\ ${ }^{3}$ Department of Rheumatology and Internal Diseases, Medical University, Bialystok, Poland \\ ${ }^{4}$ Department of Rheumatology and Rehabilitation, Medical University, Poznań, Poland \\ ${ }^{5}$ Department of Rheumatology and Connective Tissue Diseases, Medical University, Lublin, Poland \\ ${ }^{6}$ Department of Connective Tissue Disease, Institute of Rheumatology, Warsaw, Poland \\ ${ }^{7}$ Poznań Centre of Rheumatology, Śrem, Poland \\ ${ }^{8}$ Dr. Jadwiga Titz-Kosko Regional Hospital for Rheumatic Diseases, Sopot, Poland \\ ${ }^{9}$ Department of Rheumatology and Connective Tissue Diseases, Collegium Medicum, Bydgoszcz, Poland \\ ${ }^{10}$ Department of Foreign Languages, Medical University, Bialystok, Poland \\ ${ }^{11}$ School of Healthcare, University of Leeds, United Kingdom.
}

Sierakowska M, Klepacka M, Sierakowski S, Pawlak-Buś K, Leszczyński P, Majdan M, Olesińska M, Romanowski W, Bykowska-Sochacka M, Jeka S, Sierakowska J, Ndosi M, Krajewska-Kułak E. Assessment of education requirements for patients with rheumatoid arthritis, based on the Polish version of the Educational Needs Assessment Tool (Pol-ENAT), in the light of some health problems - A cross-sectional study. Ann Agric Environ Med. 2016; 23(2): 361-367. doi: 10.5604/12321966.1203906

\section{Abstract}

Introduction. Patients with chronic rheumatoid arthritis (RA) need advice in order to face the problems of everyday life, as well as suffering associated with the disease. Health professionals should attempt to raise the level of resourcefulness and independence of the patient.

Objective. To assess the relationship between the deficit of knowledge about RA and the degree of pain, fatigue, morning stiffness, assessment of disease activity as well as functional efficiency.

Materials and method. The study was conducted on 277 patients with RA in 7 rheumatologic centres in Poland. The method applied was the questionnaire Pol-ENAT (0-156); HAQ DI (0-3); analog scales (0-100).

Results. Mean (SD) age was 53.28 (13.01) and disease duration 13.70 (10.63) years. The mean (SD) value was 54.93 (23.17) for pain, 52.97 (21.98) for fatigue, 48.28 (24.76) for morning stiffness (0-100 mm). HAQ DI was $1.40(0.66)$, with an upward trend with duration of disease $(p<0.001)$.

There was a positive correlation between the demand for knowledge about the movement $(r=0.194 ; p=0.001)$, self-care $(r=0.134 ; p=0.026)$, assistance/support $(r=0.163 ; p=0.006)$ and morning stiffness experienced. There was a negative correlation between the need for knowledge concerning managing pain, feelings and the arthritis process and daily ability assessed with HAQ DI.

Conclusions. The study shows that health education should be targeted at young patients with early RA. In the case of the severity of morning joints stiffness, there is a need to increase knowledge about the methods of mobility aids, self-care and the possibility of obtaining support.

- Key words

educational needs, patients, RA, health problems

\section{INTRODUCTION}

Rheumatoid arthritis (RA) is a common, chronic inflammatory disease. If treatment of RA fails to obtain

Address for correspondence: Matylda Sierakowska, Department of Integrated Medical Care, Medical University, Maria Sklodowska-Curie 7a, 15-096 Bialystok, Poland

E-mail: matyldasierakowska@gazeta.pl

Received: 14 July 2014; accepted: 10 September 2014 remission in early disease, many patients will proceed to permanent impairment of motor function and organ damage. Each patient with RA suffers from pain, swelling, morning stiffness, fatigue, states of low spirits and depression, which all result in a lower quality of life $[1,2]$.

In Poland, it is estimated that RA affects about $1 \%$ of the population, which means that the disease causes disability in 400,000 people. The largest group affected by RA constitutes people in their forties and fifties, with the great majority being women [3]. 
Due to the fact that RA starts during the period in which the patient is most economically and socially active, the illness is difficult to accept. The diagnosis is usually a harbinger not only of chronic suffering, but also of the decrease in physical attractiveness (deformities), the need to change life plans (problems with maintaining professional and family roles), being dependent on others and general disability. The failures caused by inability to realize one's life plans sometimes might lead to escape into loneliness and embitterment, which might result in depressive disorders. The greater the severity of depressive symptoms, the less is the ability to cope with the disease $[4,5]$.

One of the main components of the complex therapeutic approach to RA is the education of patients and caretakers, and from the perspective of the latter this should be a priority task. The purpose of education, in its broad sense, is to keep the patient in his/her widest range of functioning, selfcare and self-nursing. A prerequisite for the education is identification of knowledge deficit, or the risk of hazard, and the ability of the patient/caregivers to cope with the disease, as well as motivation to participate in education. It is important to formulate measurable effects of education, as well as to plan and use of adequate methods and forms of teaching and their evaluation [6].

The effectiveness of education is determined by the active participation of a person who is educated; however, it is crucial that the objectives of the education arise from the accurate diagnosis of the real needs and expectations. The patient's education should be oriented on motivation, so that he/she would desire to positively affect his/her health [7].

In recent years, the Academic \& Clinical Unit for Musculoskeletal Nursing, University of Leeds in the UK, has developed a tool for systematically assessing the educational needs of patients with rheumatic diseases - the Educational Needs Assessment Tool (ENAT) [8]. Research in 7 European countries confirm that the ENAT is a fast and simple method of data collection targeted at the subject of education that through a detailed analysis of each domain shows which issues should be brought to the patient $[9,10]$.

To-date in Poland, there have been no studies based on a standardized research tool concerning the learning needs of patients with RA. In 2012, at the Medical University of Bialystok, in collaboration with the University of Leeds, the ENAT questionnaire was translated and subjected to validation, as well as Batton's cultural adaptation, on a group of 145 patients with RA and 133 with SSc - Scleroderma and Systemic Sclerosis [11, 12].

\section{OBJECTIVES}

The purpose of this study is to determine the educational needs of Polish patients with RA, based on the adapted version of the Polish ENAT questionnaire (Pol - ENAT) [12]., as well as analysis of the impact of socio-demographic variables on the educational needs of patients with RA about the disease. The relationship between self-reported pain, fatigue, morning stiffness of the joints, and overall assessment of the impact of the disease on daily activities, functional efficiency and the deficit of knowledge about RA and education requirements were additionally investigated.

\section{MATERIALS AND METHOD}

The study was conducted in the Department of Rheumatology and Internal Diseases at the Medical University in Bialystok, and in 6 other rheumatologic centres in Poland, on 277 patients hospitalized with rheumatoid arthritis. There were 135 patients from Bialystok, 47 from the Department of Rheumatology and Connective Tissue Diseases at the 2nd University Hospital in Bydgoszcz, 30 from the Department of Connective Tissue Disease, Institute of Rheumatology in Warsaw, 20 from the Regional Hospital for Rheumatic Diseases in Sopot, 15 from the Poznań Centre of Rheumatology in Śrem, 15 from the Department of Rheumatology and Connective Tissue Diseases at the Medical University in Lublin, and 15 from the Department of Rheumatology and Rehabilitation at the Medical University in Poznań.

The inclusion criterion for selection of the research sample was RA, based on the ACR/EULAR 2010 criteria, and age $\geq$ 18 years. The study was approved by the Bioethics Committee of the Medical University of Bialystok (R I-002-377/2012 and R-I-002/249/2014). The patients gave written consent to participate in the study.

The study used a survey method using the diagnostic technique of a patient questionnaire, filled in by each patient from June 2013 - December 2014.

Research tools were a questionnaire assessing the educational needs of Pol-ENAT $(0-156)(0=$ not at all important, 1 = little importance, 2 = fairly important, $3=$ very important, $4=$ extremely important), and the HAQ Disability Index (0-3) and visual analogue scales (0-100) that assess pain (Pain VAS), fatigue (Fatigue VAS), morning stiffness and feeling of intensity of the disease.

The Polish Needs Assessment Tool (Pol-ENAT) is a questionnaire completed by the patient. It contains 39 items, grouped into 7 domains:

1) managing pain (0-24): drugs, use of heat/cold, distraction, relaxation, exercise, acupuncture, hydrotherapy;

2) movement (0-20): using equipment, lifting, energy saving, sleep/rest, joint protection;

3) feelings (0-16): life with stress, mood/depression, fatigue;

4) arthritis process (0-28): causes, types, inheritance, medical treatment, disability;

5) treatments (0-28): demand for drugs, use, side-effects, blood tests, radiology, surgery, devices;

6) self-help measures (0-24): alternative therapies, vitamin, what to avoid, exercise, how much exercise, when to contact your doctor, nurse;

7) support systems (0-16): to help organizations, financial assistance, the group's support, recommend consultation.

Domain results are determined in values from $0-4$ Likert Scale, which equals low-high educational needs [12].

Statistical analysis. For the statistical analysis of the collected data, the method of the description and a study of the interaction between the quantitative and qualitative traits were used. For independent groups, one-way ANOVA variance to verify the hypothesis of equality of means for studied variables were used. For nonparametric data, the statistical test of independence based on Chi-square was analyzed. It was also examined whether there is a linear relationship between the measured traits. For this purpose, the authors calculated the Pearson correlation coefficient 
$r$ and Spearman's rank, as well as a test of correlation of significance, in order to verify the hypothesis of the lack of rank dependence between the studied traits. The level of significance $\alpha=0.05$. Statistical analysis was performed using PQStat v.1.4.2. and Excel.

\section{RESULTS}

A survey was conducted on 277 RA patients ( $n=277)$, including 214 women (77\%) and 63 men (23\%). Patients were divided into 3 age groups ( $\leq 40$ years, $41-60$ and $\geq 61$ ); mean (SD) age -53.28 (13.01) years. More than half of the respondents (56\%) were in the age group 41-60 years. Duration of the disease was analyzed in relation to groups $-0-5$ years, $6-10$, $11-20$, and $\geq 21$; mean duration of RA $-13.70(10.63)$ years. Most of the respondents reported secondary $(n=105,37.91 \%)$ and professional education $(\mathrm{n}=75,27.08 \%)$. Higher education was reported by 63 patients (23\%), and basic education only by $34(12 \%)$.

Analysis of analogue scales revealed that respondents' selected health problems were assessed on the mid-level. Pain VAS (0-100 mm) was 54.93 (23.17), fatigue - 52,94 (21.98), morning stiffness - 48,28 (24.76), and severity of disease 54.16 (21.33) (Tab. 1).

A detailed analysis of the obtained data, after application of Pearson's correlation coefficient ( $r$ ), indicated that age has a significant impact on the severity of the symptoms reported by the patient. There was a positive linear relationship, that proved that the older the patient, the greater the value of the assessment. This was especially noticeable in the sensation of pain (Pain VAS) $(\mathrm{r}=0.186 ; \mathrm{p}=0.002)$, morning stiffness $(\mathrm{r}=0.188 ; \mathrm{p}=0.002)$, fatigue (Fatigue-VAS $)(\mathrm{r}=0.147 ; \mathrm{p}=0.014)$ and the severity of disease $(\mathrm{r}=0.248 ; \mathrm{p}<0.001)($ Tab. 1$)$.
Patients also assessed their performance in daily activities (HAQ DI). The resulting overall average value of $1.40(0.66)$, indicated severe limitations or need for assistance in daily activities (>1-2 pts.). The results obtained on the basis of HAQ DI demonstrated that with age, 2 test groups achieved poorer performance in self-care activities $(\mathrm{r}=0.474 ; \mathrm{p}<0.001)$ (Tab. 1).

Given the variable concerning the level of education of the subjects, it was been demonstrated that there is a correlation between education and severity of the reported symptoms. Patients with higher education reported significantly fewer problems than respondents with primary or vocational education. This was observed in the perception of pain (Pain VAS) $(p=0.005)$, the presence of morning stiffness $(p=0.006)$, fatigue (Fatigue VAS) $(\mathrm{p}=0.007)$; overall disease activity influenced daily life (HAQ DI) (p<0.001) (Tab. 2).

Analysis of disease duration showed that there were significant statistical differences in the assessment of the parameters investigated (fatigue $r=0.128 ; p=0.033$ and effect of the disease on daily activity $(r=0.181 ; p=0.002)$.

An effect of the duration of the disease on progressive functional disability $(\mathrm{p}<0.001)$ was observed; patients pointed to increasing difficulty with daily activities within the period of RA development ( $0-5$ years - HAQ $0.92(0.64), \geq 21$ years - HAQ 1.91 (0.52) (Tab. 1).

Analysis of the Pol - ENAT questionnaire. The great majority of respondents $(n=250 ; 90 \%)$ indicated that they would like to have information that may help in coping with the disease, whereas as much as $40 \%$ of respondents $(\mathrm{n}=111)$, according to the introductory evaluation, needed to know everything. Overall assessment of the educational needs of respondents, on a scale of $0-156$, identified the deficit of knowledge at the secondary level (information

Table 1. Effect of age and disease duration on values of feeling some clinical symptomsand functional disability index (HAQ DI) of RA patients

\begin{tabular}{|c|c|c|c|c|c|c|c|c|c|c|}
\hline \multicolumn{2}{|c|}{ STUDIED VARIABLES } & \multicolumn{4}{|c|}{$\begin{array}{l}\text { AGE, years Mean }(S D) \\
53.28(13.01)\end{array}$} & \multicolumn{5}{|c|}{$\begin{array}{l}\text { DISEASE DURATION years Mean (SD) } \\
13.70(10.63)\end{array}$} \\
\hline Score range & $\begin{array}{c}\text { Total } \\
\text { Mean (SD) }\end{array}$ & $\begin{array}{c}\leq 40 \\
n=46\end{array}$ & $\begin{array}{l}41-60 \\
n=155\end{array}$ & $\begin{array}{l}>60 \\
n=76\end{array}$ & ${ }_{p}^{1} r_{p}$ & $\begin{array}{c}0-5 \\
n=59\end{array}$ & $\begin{array}{l}6-10 \\
n=80\end{array}$ & $\begin{array}{l}11-20 \\
n=86\end{array}$ & $\begin{array}{l}\geq 21 \\
\mathrm{n}=52\end{array}$ & ${ }^{1} r_{p}$ \\
\hline \multirow{2}{*}{$\begin{array}{l}\text { Pain-VAS } \\
(0-100)\end{array}$} & \multirow{2}{*}{$54.93(23.17)$} & $\begin{array}{c}50.13 \\
(24.33) \\
\end{array}$ & $\begin{array}{c}52.72 \\
(23.70) \\
\end{array}$ & $\begin{array}{c}62.33 \\
(19.67) \\
\end{array}$ & \multirow{2}{*}{$\begin{array}{c}0.186 \\
(0.002)\end{array}$} & \multirow{2}{*}{$\begin{array}{c}55.80 \\
(24.97)\end{array}$} & \multirow{2}{*}{$\begin{array}{c}50.69 \\
(22.36)\end{array}$} & \multirow{2}{*}{$\begin{array}{c}56.17 \\
(22.78)\end{array}$} & \multirow{2}{*}{$\begin{array}{c}58.40 \\
(22.63)\end{array}$} & \multirow{2}{*}{$\begin{array}{c}0.091 \\
(0.129)\end{array}$} \\
\hline & & & $\begin{array}{l}\text { 5. }>60^{2} p= \\
\text { vs. }>60 \quad p\end{array}$ & & & & & & & \\
\hline \multirow{2}{*}{$\begin{array}{l}\text { Fatigue-VAS } \\
(0-100)\end{array}$} & \multirow{2}{*}{$\begin{array}{c}52.94 \\
(21.98)\end{array}$} & $\begin{array}{c}50.96 \\
(22.09) \\
\end{array}$ & $\begin{array}{c}50.78 \\
(22.03) \\
\end{array}$ & $\begin{array}{c}58.67 \\
(21.05) \\
\end{array}$ & \multirow{2}{*}{$\begin{array}{c}0.147 \\
(0.014)\end{array}$} & \multirow{2}{*}{$\begin{array}{c}49.72 \\
(22.81)\end{array}$} & \multirow{2}{*}{$\begin{array}{c}50.24 \\
(22.60)\end{array}$} & \multirow{2}{*}{$\begin{array}{c}55.18 \\
(20.30)\end{array}$} & \multirow{2}{*}{$\begin{array}{l}57.23 \\
(22.21)\end{array}$} & \multirow{2}{*}{$\begin{array}{c}0.128 \\
(0.033)\end{array}$} \\
\hline & & & vs. $>60$ & & & & & & & \\
\hline \multirow{2}{*}{$\begin{array}{l}\text { Severity of disease } \\
(0-100)\end{array}$} & \multirow{2}{*}{$\begin{array}{c}54.16 \\
(21.33)\end{array}$} & $\begin{array}{c}45.69 \\
(21.14)\end{array}$ & $\begin{array}{c}52.79 \\
(21.54)\end{array}$ & $\begin{array}{c}62.06 \\
(18.50)\end{array}$ & \multirow{2}{*}{$\begin{array}{c}0.248 \\
(<0.001)\end{array}$} & $\begin{array}{l}49.25 \\
(22.60)\end{array}$ & $\begin{array}{l}52.06 \\
(22.30)\end{array}$ & $\begin{array}{l}54.20 \\
(20.54)\end{array}$ & $\begin{array}{c}62.87 \\
(20.66)\end{array}$ & \multirow{2}{*}{$\begin{array}{c}0.181 \\
(0.002)\end{array}$} \\
\hline & & \multicolumn{3}{|c|}{$\begin{array}{c}\leq 40 \text { vs. }>60 p<0.001 \\
41-60 \text { vs. }>60 p=0.004\end{array}$} & & \multicolumn{4}{|c|}{$\begin{array}{l}0-5 \text { vs. } \geq 21 p=0.002 \\
6-10 \text { vs. } \geq 21 p=0.004 \\
11-20 \text { vs. } \geq 21 p=0.02\end{array}$} & \\
\hline \multirow{2}{*}{$\begin{array}{l}\text { Morning stiffness } \\
(0-100)\end{array}$} & \multirow[t]{2}{*}{$48.28(24.76)$} & $\begin{array}{c}39.63 \\
(21.77) \\
\end{array}$ & $\begin{array}{c}47.44 \\
(24.71) \\
\end{array}$ & $\begin{array}{c}55.24 \\
(24.93) \\
\end{array}$ & \multirow{2}{*}{$\begin{array}{c}0.188 \\
(0.002)\end{array}$} & \multirow{2}{*}{$\begin{array}{c}44.31 \\
(23.39)\end{array}$} & \multirow{2}{*}{$\begin{array}{c}49.08 \\
(24.08)\end{array}$} & \multirow{2}{*}{$\begin{array}{c}48.64 \\
(25.06)\end{array}$} & \multirow{2}{*}{$\begin{array}{c}50.99 \\
(26.91)\end{array}$} & \multirow{2}{*}{$\begin{array}{c}0.098 \\
(0.104)\end{array}$} \\
\hline & & & s. $>60 p=$ & & & & & & & \\
\hline \multirow{2}{*}{$\begin{array}{l}\text { HAQ DI } \\
(0-3)\end{array}$} & \multirow{2}{*}{$\begin{array}{c}1.40 \\
(0.66)\end{array}$} & $\begin{array}{c}0.87 \\
(0.58)\end{array}$ & $\begin{array}{c}1.37 \\
(0.62)\end{array}$ & $\begin{array}{c}1.80 \\
(0.53)\end{array}$ & \multirow{2}{*}{$\begin{array}{c}0.474 \\
(<0.001)\end{array}$} & $\begin{array}{c}0.92 \\
(0.64)\end{array}$ & $\begin{array}{c}1.33 \\
(0.56)\end{array}$ & $\begin{array}{c}1.49 \\
(0.60)\end{array}$ & $\begin{array}{c}1.91 \\
(0.52)\end{array}$ & \multirow{2}{*}{$\begin{array}{c}0.442 \\
(<0.001\end{array}$} \\
\hline & & $\leq 40$ vs. $>60$ & $\begin{array}{l}0 \text { vs. } 41-6 \\
p<0.001\end{array}$ & 60 vs. $>60$ & & \multicolumn{4}{|c|}{$\begin{array}{l}0-5 \text { vs. } 6-10 ; 0-5 \text { vs. } 11-20 \\
\quad 0-5 \text { vs. } \geq 21 \mathrm{p}<0.001 \\
0 \text { vs. } \geq 21 ; 11-20 \text { vs. } \geq 21 \mathrm{p}<0.001\end{array}$} & \\
\hline
\end{tabular}

Pearson's correlation coefficient $r$

${ }^{2}$ univariate ANOVA for independent groups 
Table 2. The effect of the educational background on the values of feeling some clinical symptomsand functional disability index (HAQ) of RA patients

\begin{tabular}{|c|c|c|c|c|c|}
\hline STUDIED VARIABLES & & EDUCATION & LBACKGROUN & DMean (SD & \\
\hline (score range) & $\begin{array}{c}\text { Basic I } \\
\mathrm{n}=34\end{array}$ & $\begin{array}{c}\text { Professional } \\
\quad \| \quad n=75\end{array}$ & $\begin{array}{c}\text { Secondary III } \\
n=105\end{array}$ & $\begin{array}{c}\text { Above IV } \\
n=63\end{array}$ & ${ }^{2} p$-value \\
\hline Pain-VAS (0-100) & $\begin{array}{c}64.97 \\
(15.95)\end{array}$ & $\begin{array}{c}56.46 \\
(24.70) \\
\end{array}$ & $54.79(21.10)$ & $\begin{array}{c}47.92 \\
(25.91)\end{array}$ & 0.005 \\
\hline & & | vs. I & $p=0.003$ & & \\
\hline Fatiaue-У/A S $(0-100)$ & $\begin{array}{c}60.76 \\
(18.18)\end{array}$ & $\begin{array}{l}55.89 \\
(22.35)\end{array}$ & $52.49(21.23)$ & $\begin{array}{l}46.10 \\
(23.02)\end{array}$ & 1007 \\
\hline & & vs. IV $p=0.009$ & II vs. IV $p=0$ & 042 & \\
\hline Severity of disease & $\begin{array}{c}64.53 \\
(14.62)\end{array}$ & $\begin{array}{l}56.93 \\
(22.24)\end{array}$ & $\begin{array}{l}53.80 \\
(20.17)\end{array}$ & $\begin{array}{c}45.85 \\
(22.34)\end{array}$ & $<0.001$ \\
\hline & & vs. IV $p=0.009$ & II vs. IV $p=0$ & 042 & \\
\hline Morning stiffness & $\begin{array}{c}55.85 \\
(25.77)\end{array}$ & $\begin{array}{l}53.49 \\
(24.17)\end{array}$ & $46.44(23.16)$ & $\begin{array}{c}41.08 \\
(25.62)\end{array}$ & \\
\hline$(0-100)$ & I vs & III $p=0.045, \quad$ । & $\begin{array}{l}\text { s. IV } p<0.001 \\
0.010\end{array}$ & II vs. IV & \\
\hline & $\begin{array}{c}1.87 \\
(0.58)\end{array}$ & $\begin{array}{c}1.46 \\
(0.64)\end{array}$ & $\begin{array}{c}1.38 \\
(0.62)\end{array}$ & $\begin{array}{c}1.11 \\
(0.65)\end{array}$ & \\
\hline & & $\begin{array}{l}\text { II } p=0.010 \\
\text { I vs. IV } p=0.00\end{array}$ & $\begin{array}{l}\text { s. IIII I vs. IV p } \\
\text { III vs. IV } p=C\end{array}$ & $\begin{array}{l}0.001 \\
028\end{array}$ & \\
\hline
\end{tabular}

${ }^{2}$ the univariate ANOVA for independent groups fairly important). The greatest demand was observed in the domain on the methods of pharmacological and nonpharmacological pain management (11.82, SD 4.97), self-care (10.99, SD 5.16) and feelings (7.61, SD 4.34) (Tab. 3).

Detailed analysis of the results of the Pol-ENAT questionnaire indicated that independent variables, such as age and gender, had an impact on the educational needs of patients. Women want to know significantly more about dealing with emotions (7.94, SD 4.37) than men (6.46, SD 4.07) $(\mathrm{p}=0.017)$. There was also a significantly higher need for respondents aged $\leq 40$ years, in all domains of the PolENAT: emotions, course of the disease $(p<0.001)$, coping with pain $(\mathrm{p}=0.001)$, self-care treatment $(\mathrm{p}=0.002)$ and treatment $(\mathrm{p}=0.029)$; however, there were no significant differences in the mobility domain. General education also affected the patients' interest in education. The study indicated that the lowest need for education, in all domains of Pol-ENAT questionnaire, were stated by the respondents with primary education, while the highest need for education was stated by patients with average education ( $\mathrm{p}=0.004)$; the values of the domains were: arthritis process and treatments $(\mathrm{p}=0.006)$, movement $(\mathrm{p}=0.014)$, feelings $(\mathrm{p}=0.036)$ and managing pain $(\mathrm{p}=0.037)($ Tab. 3).

Given the variable duration of the disease, in subjects with early RA there was a significantly higher need for education in the domain of coping with emotions (stress associated with

Table 3. The effect of the age and educational background on the needs for education using Pol-ENAT questionnaire of RA patients

\begin{tabular}{|c|c|c|c|c|c|c|c|c|c|}
\hline \multirow[t]{2}{*}{ Pol-ENAT } & \multicolumn{4}{|c|}{ AGEMean (SD) } & \multicolumn{5}{|c|}{ EDUCATIONAL BACKGROUNDMean (SD) } \\
\hline & $\leq 40$ & $41-60$ & $>60$ & ${ }^{2} p$-value & Basic I & Professional II & Secondary III & Above IV & ${ }^{2} p$-value \\
\hline $\begin{array}{r}\text { TOTAL } \\
(0-156)\end{array}$ & $\begin{array}{l}74.97 \\
(7.60)\end{array}$ & $\begin{array}{c}67.68 \\
(28.41)\end{array}$ & $\begin{array}{c}58.65 \\
(30.61)\end{array}$ & \multirow{2}{*}{0.008} & $\begin{array}{c}51.23 \\
(24.08)\end{array}$ & $\begin{array}{c}70.29 \\
(26.94)\end{array}$ & $\begin{array}{c}70.37 \\
(32.05)\end{array}$ & $\begin{array}{c}63.40 \\
(27.35)\end{array}$ & \multirow{2}{*}{0.004} \\
\hline Mean (SD) & \multicolumn{3}{|c|}{$\leq 40$ vs. $>60 \quad p=0.008$} & & \multicolumn{4}{|c|}{ I vs. II $p=0.003$ I vs. III $p=0.005$} & \\
\hline \multirow{2}{*}{$\begin{array}{l}\text { Managingpain } \\
(0-24) \\
11.82(4.97)\end{array}$} & $\begin{array}{l}13.47 \\
(5.16)\end{array}$ & $\begin{array}{l}12.13 \\
(4.61)\end{array}$ & $\begin{array}{l}10.20 \\
(5.17)\end{array}$ & \multirow{2}{*}{0.001} & $\begin{array}{c}9.61 \\
(4.02)\end{array}$ & $\begin{array}{l}11.99 \\
(4.21)\end{array}$ & $\begin{array}{l}12.43 \\
(5.11)\end{array}$ & $\begin{array}{l}11.79 \\
(5.74)\end{array}$ & \multirow{2}{*}{0.037} \\
\hline & \multicolumn{3}{|c|}{$\begin{array}{cc}\leq 40 \text { vs. }>60 & p=0.001 \\
41-60 \text { vs. }>60 & p=0.013\end{array}$} & & \multicolumn{4}{|c|}{ I vs. III $p=0.020$} & \\
\hline \multirow{2}{*}{$\begin{array}{l}\text { Movement } \\
(0-20) \\
7.93(4.10)\end{array}$} & \multirow{2}{*}{$\begin{array}{l}8.06 \\
(3.98)\end{array}$} & \multirow[t]{2}{*}{$8.14(4.01)$} & \multirow[t]{2}{*}{$7.42(4.37)$} & \multirow[t]{2}{*}{0.44} & $\begin{array}{c}6.78 \\
(3.76)\end{array}$ & $\begin{array}{l}8.55 \\
(3.83)\end{array}$ & $\begin{array}{c}8.49 \\
(4.33)\end{array}$ & $\begin{array}{c}6.89 \\
(3.96)\end{array}$ & \multirow[t]{2}{*}{0.014} \\
\hline & & & & & \multicolumn{4}{|c|}{ II vs. IV ${ }^{2} p=0.046$} & \\
\hline $\begin{array}{l}\text { Feelings } \\
(0-16) \\
7.61(4.34)\end{array}$ & $\begin{array}{c}9.68 \\
(4.33)\end{array}$ & $\begin{array}{l}7.62 \\
(4.20)\end{array}$ & $\begin{array}{c}6.33 \\
(4.20)\end{array}$ & $<0.001$ & $\begin{array}{c}5.58 \\
(3.46)\end{array}$ & $\begin{array}{c}7.95 \\
(4.18)\end{array}$ & 7.89 & $\begin{array}{l}7.81 \\
(4.55)\end{array}$ & 0.036 \\
\hline \multirow{2}{*}{$\begin{array}{l}\text { Arthritisprocess } \\
(0-28) \\
10.63(6.50)\end{array}$} & $\begin{array}{l}13.80 \\
(6.99)\end{array}$ & $\begin{array}{l}10.99 \\
(6.18)\end{array}$ & $\begin{array}{c}7.97 \\
(5.85)\end{array}$ & \multirow[b]{2}{*}{$<0.001$} & $\begin{array}{c}7.36 \\
(4.37)\end{array}$ & $\begin{array}{l}10.73 \\
(6.04)\end{array}$ & $\begin{array}{l}11.83 \\
(7.20)\end{array}$ & $\begin{array}{l}10.28 \\
(6.26)\end{array}$ & \multirow[b]{2}{*}{0.006} \\
\hline & & $\begin{array}{l}>41-60 p \\
\text { s. }>60 p< \\
\text { vs. }>60 \quad p=\end{array}$ & $\begin{array}{l}.021 \\
01 \\
002\end{array}$ & & & I vs. III & $=0.003$ & & \\
\hline \multirow{2}{*}{$\begin{array}{l}\text { Treatments } \\
(0-28) \\
11.01(6.49)\end{array}$} & $\begin{array}{l}12.25 \\
(5.64)\end{array}$ & $\begin{array}{l}11.43 \\
(6.36)\end{array}$ & $\begin{array}{c}9.39 \\
(7.00)\end{array}$ & \multirow{2}{*}{0.029} & $\begin{array}{c}8.65 \\
(6.20)\end{array}$ & $\begin{array}{l}12.03 \\
(5.63)\end{array}$ & $\begin{array}{l}11.96 \\
(7.34)\end{array}$ & $\begin{array}{c}9.48 \\
(5.54)\end{array}$ & \multirow{2}{*}{0.006} \\
\hline & & 's. $>60 p=$ & & & & | vs. III & $=0.045$ & & \\
\hline \multirow{2}{*}{$\begin{array}{l}\text { Self-helpmeasures } \\
(0-24) \\
10.99(5.16)\end{array}$} & $\begin{array}{l}11.72 \\
(4.18)\end{array}$ & $\begin{array}{l}11.62 \\
(5.34)\end{array}$ & $\begin{array}{c}9.26 \\
(4.96)\end{array}$ & \multirow{2}{*}{0.002} & $\begin{array}{c}8.79 \\
(4.46)\end{array}$ & $\begin{array}{l}11.69 \\
(5.25)\end{array}$ & $\begin{array}{l}11.18 \\
(4.97)\end{array}$ & $\begin{array}{l}11.02 \\
(4.97)\end{array}$ & \multirow{2}{*}{0.051} \\
\hline & & $\begin{array}{l}\text { s. }>60 p= \\
\text { vs. }>60 \quad p=\end{array}$ & $\begin{array}{l}27 \\
003\end{array}$ & & & | vs. II & $=0.032$ & & \\
\hline $\begin{array}{l}(0-16) \\
5.45(3.89)\end{array}$ & $(3.90)$ & $(3.90)$ & (3.79) & 0.059 & & II vs. IV & $=0.044$ & & 0.023 \\
\hline
\end{tabular}


Table 4. The effect of the disease durationon the domains of the questionnaire Pol-ENAT

\begin{tabular}{|c|c|c|c|c|c|c|}
\hline \multirow[t]{2}{*}{ Pol-ENAT } & \multicolumn{6}{|c|}{ DISEASE DURATION years Mean (SD) } \\
\hline & $0-5$ & $6-10$ & $11-20$ & $\geq 21$ & $\begin{array}{c}\text { 2pp } \\
\text { value }\end{array}$ & $\underset{{ }^{1} r_{p}}{P \text {-value }}$ \\
\hline $\begin{array}{r}\text { TOTAL } \\
(0-156)\end{array}$ & $\begin{array}{c}77.06 \\
(31.40)\end{array}$ & $\begin{array}{c}67.81 \\
(27.18)\end{array}$ & $\begin{array}{c}59.36 \\
(23.56)\end{array}$ & $\begin{array}{c}63.83 \\
(35.17)\end{array}$ & \multirow{2}{*}{0.003} & \multirow{2}{*}{$\begin{array}{c}-0.226 \\
(<0.001)\end{array}$} \\
\hline DOMAINS & \multicolumn{4}{|c|}{$\begin{array}{r}0-5 \text { vs. } 11-20 \quad p<0.001, \\
p=0.02\end{array}$} & & \\
\hline \multirow{2}{*}{$\begin{array}{l}\text { Managing pain } \\
(0-24)\end{array}$} & $\begin{array}{l}13.64 \\
(5.68)\end{array}$ & $\begin{array}{l}12.17 \\
(4.40)\end{array}$ & $\begin{array}{l}10.77 \\
(4.31)\end{array}$ & $\begin{array}{l}10.96 \\
(5.41)\end{array}$ & \multirow{2}{*}{0.003} & \multirow{2}{*}{$\begin{array}{l}-0.226 \\
(<0.001)\end{array}$} \\
\hline & \multicolumn{4}{|c|}{$\begin{array}{r}0-5 \text { vs. } 11-20 \quad p<0.001 \\
p=0.004\end{array}$} & & \\
\hline $\begin{array}{l}\text { Movement } \\
(0-20)\end{array}$ & $\begin{array}{c}8.44 \\
(4.47) \\
\end{array}$ & $\begin{array}{c}7.94 \\
(3.87)\end{array}$ & $\begin{array}{c}7.45 \\
(3.08)\end{array}$ & $\begin{array}{c}8.13 \\
(5.36)\end{array}$ & 0.53 & $\begin{array}{l}-0.038 \\
(0.529)\end{array}$ \\
\hline \multirow{2}{*}{$\begin{array}{l}\text { Feelings } \\
(0-16)\end{array}$} & $\begin{array}{c}9.75 \\
(4.46)\end{array}$ & $\begin{array}{c}7.24 \\
(3.98)\end{array}$ & $\begin{array}{c}6.80 \\
(4.03)\end{array}$ & $\begin{array}{c}7.07 \\
(4.56)\end{array}$ & \multirow{2}{*}{$<0.001$} & \multirow{2}{*}{$\begin{array}{c}-0.198 \\
(<0.001)\end{array}$} \\
\hline & \multicolumn{4}{|c|}{$\begin{array}{l}0-5 \text { vs. } 6-10 ; 0-5 \text { vs. } 11-20 ; 0-5 \text { vs. } \geq 21 \\
\qquad p=0.001\end{array}$} & & \\
\hline \multirow{2}{*}{$\begin{array}{l}\text { Arthritis process } \\
(0-28)\end{array}$} & $\begin{array}{l}12.46 \\
(7.19)\end{array}$ & $\begin{array}{l}11.68 \\
(6.59)\end{array}$ & $\begin{array}{c}9.10 \\
(4.76)\end{array}$ & $\begin{array}{c}9.47 \\
(7.36)\end{array}$ & \multirow[b]{2}{*}{0.004} & \multirow[b]{2}{*}{$\begin{array}{c}-0.213 \\
(<0.001)\end{array}$} \\
\hline & \multicolumn{4}{|c|}{$\begin{array}{c}0-5 \text { vs. } 11-20 p=0.002 \\
0-5 \text { vs. } \geq 21 ; 6-10 \text { vs. } 11-20 \quad p=0.01 \\
6-10 \text { vs. } \geq 21 p=0.05\end{array}$} & & \\
\hline \multirow{2}{*}{$\begin{array}{l}\text { Treatments } \\
(0-28)\end{array}$} & $\begin{array}{l}12.69 \\
(7.13)\end{array}$ & $\begin{array}{l}11.39 \\
(5.72)\end{array}$ & $\begin{array}{c}9.59 \\
(5.56)\end{array}$ & $\begin{array}{l}10.88 \\
(7.82)\end{array}$ & \multirow[t]{2}{*}{0.04} & \multirow{2}{*}{$\begin{array}{l}-0.150 \\
(0.012)\end{array}$} \\
\hline & \multicolumn{4}{|c|}{$0-5$ vs. $11-20 p=0.005$} & & \\
\hline \multirow[b]{2}{*}{$\begin{array}{l}\text { Self-help } \\
\text { measures } \\
(0-24)\end{array}$} & $\begin{array}{l}12.52 \\
(5.44)\end{array}$ & $\begin{array}{l}11.75 \\
(5.16)\end{array}$ & $\begin{array}{c}9.85 \\
(4.28)\end{array}$ & $\begin{array}{c}9.96 \\
(5.62)\end{array}$ & \multirow[b]{2}{*}{0.004} & \multirow[b]{2}{*}{$\begin{array}{c}-0.199 \\
(<0.001)\end{array}$} \\
\hline & \multicolumn{4}{|c|}{$\begin{array}{c}0-5 \text { vs. } 11-20 p=0.002,0-5 \text { vs. } \geq 21 \\
p=0.008\end{array}$} & & \\
\hline $\begin{array}{l}\text { Support } \\
\text { systems } \\
(0-16)\end{array}$ & $\begin{array}{c}5.92 \\
(4.52)\end{array}$ & $\begin{array}{c}5.63 \\
(3.62)\end{array}$ & $\begin{array}{c}4.93 \\
(3.07)\end{array}$ & $\begin{array}{c}5.52 \\
(4.70)\end{array}$ & 0.46 & $\begin{array}{l}-0.072 \\
(0.233)\end{array}$ \\
\hline
\end{tabular}

${ }^{2}$ the univariate ANOVA for independent groups

the disease, mood/depression, fatigue) than in the patients in the later phase of the disease $(\mathrm{p}<0.001)$. Respondents with disease duration of $0-5$ years also reported higher educational needs concerning issues related to coping with pain $(p=0.003)$, arthritis process $(p=0.004)$, methods of therapy that can be used by itself (self-care) $(p=0.004)$, as well as treatment $(\mathrm{p}=0.04)$ (Tab. 4).

Analysis of educational needs in relation to pain, morning stiffness, fatigue, intensity of disease andpatients' disability was performed by the means of looking for a correlation between the variables. There was a positive correlation between the demand for knowledge concerning movement and the higher assessment of the impact of the disease on daily activity $(\mathrm{r}=0.122, \mathrm{p}=0.043)$. The study also indicated that patients who suffer from a high severity of morning stiffness significantly more often declared the need to broaden their knowledge. There was a positive correlation concerning the severity of morning stiffness for education in the field of movement $(\mathrm{r}=0.194 ; \mathrm{p}=0.001)$, self-care methods $(\mathrm{r}=0.134$; $\mathrm{P}=0.026)$, and support services/aid $(\mathrm{r}=0.163 ; \mathrm{p}=0.006)$ (Tab. 5).

However, there were no positive correlations between disability in daily activities (HAQ) and greater demand for education. There was a negative correlation between the increase of HAQ and the lesser need for education, especially in the Pol-ENAT domains of managing pain $(\mathrm{r}=-0.175$; $\mathrm{p}=0.003)$, feelings $(\mathrm{r}=-0 ; 147, \mathrm{p}=0.014)$ and inflammatory process $(\mathrm{r}=-0.147 ; \mathrm{p}=0.014)$ (Tab. 5).

\section{DISCUSSION}

In the course of chronic, progressive RA, there is impairment of the body's biological functions, functional disability, mental disorders and changes in the performance of social roles and professions. Recommendations for treatment (ACR/EULAR 2010) clearly define the goal - long-term maintenance of the best possible quality of life of the patient by controlling the symptoms of the disease, pain relief, prevention of destructive changes in the joints, normalization of physical function and providing help in social participation [13]. This results in the need for making large-scale educational activities in order to prepare the patients for non-professional care and self-control of their own health [14].

In accordance with the recommendations of EULAR, the role of a nurse is the therapeutic education of RA patients, aimed at improving the strategies of coping with the disease and increasing the capacity for self-care [15].

Table 5. The analysis of correlation between the domains of Pol-ENAT and the values of feeling some clinical symptomsand functional disability index (HAQ) of RA patients

\begin{tabular}{|c|c|c|c|c|c|c|c|c|}
\hline \multirow[b]{2}{*}{$\begin{array}{l}\text { STUDIED VARIABLES } \\
\text { (score range) }\end{array}$} & & \multicolumn{7}{|c|}{ Pol-ENAT domains (0-156) Mean (SD) $66.41 \pm 29.32$} \\
\hline & & Managing pain & Movement & Feelings & Arthritisproces & Treatments & Self-helpmeasure & Supportsystems \\
\hline $\begin{array}{l}\text { Pain-VAS } \\
(0-100)\end{array}$ & p-value & 0.665 & 0.108 & 0.573 & 0.180 & 0.353 & 0.745 & 0.644 \\
\hline $\begin{array}{l}\text { Fatigue-VAS } \\
(0-100)\end{array}$ & $r_{p}$ & -0.005 & 0.109 & 0.061 & -0.024 & 0.010 & 0.026 & 0.022 \\
\hline \multirow{2}{*}{$\begin{array}{l}\text { Severity of disease } \\
(0-100)\end{array}$} & $r_{p}$ & -0.052 & 0.122 & 0.010 & -0.081 & -0.037 & 0.015 & 0.025 \\
\hline & $\mathrm{p}$-value & 0.389 & 0.043 & 0.870 & 0.175 & 0.532 & 0.794 & 0.682 \\
\hline \multirow{2}{*}{$\begin{array}{l}\text { Morningstiffness } \\
(0-100)\end{array}$} & $r_{p}$ & 0.048 & 0.194 & 0.075 & 0.030 & 0.039 & 0.134 & 0.163 \\
\hline & p-value & 0.428 & 0.001 & 0.212 & 0.613 & 0.515 & 0.026 & 0.006 \\
\hline $\begin{array}{l}\text { HAQ DI } \\
(0-3)\end{array}$ & $r_{p}$ & -0.175 & 0.116 & -0.147 & -0.147 & -0.101 & -0.082 & 0.046 \\
\hline
\end{tabular}

'Pearson's correlationcoefficient $r$ 
The aim of the study was to identify the educational needs of patients in the context of their health problems, as expressed in their subjective assessment.

The assessment of the health parameters of the respondents $(\mathrm{n}=277)$, such as pain, morning stiffness, fatigue, general feeling of the impact of the disease on functioning in everyday life, based on a linear scale (0-100), indicated that the subjective assessment of above- mentioned problems was on the moderate level. Pain VAS was 54.93 and the sense of the impact of the disease on daily activities - 54.16. These values are somewhat higher than fatigue -52.94 and morning stiffness - 48.29.

The progressive nature of the disease makes patients' functioning worse in daily activities. HAQ disability index (0-3) allows the assessment of the everyday performance. The value of HAQ $\geq 1$ signifies the exponent of clinical disability [16].

In the presented study, patients assessed their performance in daily activities (HAQ DI) at 1.40. It was also observed that the duration of the disease had an impact on the progressive lack of functional capacity $(\mathrm{p}<0.001)$. Patients pointed to increasing difficulty in daily activities with the period of RA duration. According to Ambriz Murillo et al., patients with early RA (mean disease duration 5.57) showed moderate assessment of disability - HAQ of 0.82 [17]. The current analysis showed that patients with disease duration of $0-5$ years obtained a lower value of the Index malfunction of HAQ -0.92 , than in the period of $6-10$ years -1.33 . Other Polish studies of patients with RA showed higher mean HAQ DI, regardless of the type of medication - patients treated with DMARDs - HAQ 1.3, and biological treatment - HAQ 1.4 [18].

According to a multicentre Dutch study, patients' expectations and needs in relation to rheumatological nursing care is mainly focused on education, promotion of self-care, well-organized care and emotional support [19].

The presented study, based on the Polish version of the Pol-ENAT questionnaire, indicates that the great majority of respondents $(n=250 ; 90 \%)$ would like to have information that may help them in coping with the disease, and as many as $40 \%$ of patients $(n=111)$ would like to know everything. Similarly, studies using the Austrian version of the ENAT (OENAT) confirm the high interest in the education of RA patients [20]. These results are consistent with previous studies in patients with RA and patients with other rheumatic diseases that indicate a high demand for information on diseases and medical care $[21,22]$.

Evaluation of the different domains of the Pol-ENAT questionnaire on a scale of $0-156$, indicated that the greatest needs of Polish patients were declared in the domain of methods of coping with pain (11.82), self-care (10.99) and feelings (7.61). Dutch researchers, who used the Dutch version of the Educational Needs Assessment (DENAT), observed partly different results. They indicate that Dutch patients $(n=165)$ expected more information in the field of the arthritis process, treatments from health professionals and self-help measures [23].

In the current study there was an attempt to examine the extent to which the severity of health problems might affect the educational needs of patients. Analyses showed that patients who suffered from a high severity of morning stiffness were significantly more likely to declare the need for knowledge in the field of mobility $(r=0.194 ; p=0.001)$, methods of self- care $(r=0.134 ; p=0.026)$ and support services/help $(r=0.163$; $\mathrm{p}=0.006$ ). There was also the need for knowledge concerning the movement in patients who evaluated higher the effect of the disease on their daily activities $(\mathrm{r}=0.122 ; \mathrm{p}=0.043)$.

The main psychological problem of patients suffering from RA is anxiety resulting from the recurrent inflammation, and which may arise as a consequence of pain. Patients are afraid that the pain will return, will be worse or persistent [4]. A relatively higher incidence of depression and anxiety in patients with RA was also reported [24].

An Austrian-German Educational Needs Assessment study (OENAT), indicated a fairly strong interest of the patients, especially women, in topics related to emotions [20]. The educational needs of their own work in coping with emotions $(0-16)$ were rated as quite important (7.61). Detailed analysis of the results of the Pol-ENAT questionnaire indicates that the independent variables, such as gender, had an impact on the educational needs of the respondents in the above domain. Women wanted to know significantly more about dealing with emotions than men $(\mathrm{p}=0.017)$.

Evaluation of the impact of age on the educational needs indicated that younger patients had greater needs in this area. This is confirmed by the study based on the DENAT questionnaire, where younger patients had a deficit of knowledge in domains related to coping with pain, emotion, and obtaining support, which is higher than in older patients [23]. Polish observations also indicate significantly higher education needs at the age of $\leq 40(\mathrm{p}=0.008)$, and in almost all domains in the Pol-ENAT questionnaire, but with no significant differences in the mobility domain.

A review of the literature regarding the impact of education on the level of knowledge about the disease indicates that the interest in information about RA is higher among both women and men with higher education [25]. The findings of the current study show that respondents with secondary and vocational education declared significantly more educational needs $(\mathrm{p}=0.004)$ in almost all domains of the Pol-ENAT questionnaire (with the exception of the domain on selfcare) than respondents with primary education or higher. Research based on the DENAT and other smaller studies confirm that interest in education is smaller with the duration of the disease, which goes hand-in-hand with progressive functional disability [21, 22, 23]. In the current study, it was observed that the higher the level of disability (HAQ $\mathrm{DI})$, the less the need for education, especially in domains such as coping with pain $(\mathrm{r}=-0.175 ; \mathrm{p}=0.003)$, emotion and inflammatory processes $(r=-0.147 ; \mathrm{p}=0.014)$.

It should be noted that the implemented treatment in patients with chronic rheumatic disease bring benefit to a greater or lesser extent; however, it requires time and commitment, both from the patient and health professionals. Nurses should devise effective methods and forms of educational interactions, with greater emphasis placed on group forms and methods of using information technology and the Internet [26]. The objectives and content of education should be jointly planned with the patient, in accordance with the recommendations of EULAR education, and designed according to specific needs $[7,14]$. 


\section{CONCLUSIONS}

Together with the duration of the disease there is a significant decrease in the level of functional efficiency, whereas the demand for knowledge is reduced. Research shows that education should be targeted at young patients with early RA, particularly in the course and specificity of coping with pain, self-care, feelings and the inflammatory process.

An important issue for women is coping with emotions. This indicates a need for assistance/ psychological consultation.

Although patients with primary education suffer from severe symptoms associated with the disease, they are not interested in health education. This group should be subjected to special care and support.

In the case of the severity of morning joints stiffness, there is a need to increase knowledge about the methods of mobility aids (in cooperation with a physiotherapist), self-care and information about the possibility of obtaining assistance/support.

\section{Acknowledgements}

This study was supported by a research project from the Medical University of Bialystok, Poland (UMB 143-10882P).

\section{REFERENCES}

1. Aletaha D, Neogi T, Silman AJ, et al. Rheumatoid Arthritis Classification Criteria An American College of Rheumatology/European League Against Rheumatism Collaborative Initiative. Arthritis Rheum. 2010; 62, 9: 2569-2581, doi: 10.1002/art.27584.

2. Głuszko P, Filipowicz-Sosnowska A, Tłustochowicz W. Rheumatoid arthritis. Recommendations for diagnosis and treatment. [Reumatoidalne zapalenie stawów. Zalecenia postepowanie diagnostycznego i terapeutycznego]. Reumatologia 2012; 50(2): 83-90.

3. Wiland P, Maciążek-Chyra B. Situation of rheumatology in Poland in 2012. [Stan reumatologii w Polsce w 2012 roku]. Reumatologia 2012; 50(4): 263-275.

4. Newth S, Delongis A. Individual differences, mood and coping with chronic pain in rheumatoid arthritis: a daily process analysis. Psychol Health. 2004; 19: 283-305.

5. Sierakowska M, Krajewska-Kułak E, Sierakowski S. Progressive physical disability, pain and quality of life of patients with rheumatoid inflammation of joints.[Postępująca niesprawność, ból i jakość życia pacjentów z reumatoidalnym zapaleniem stawów]. Przegl Lek. 2005; 62(2): 89-94.

6. Sierakowska M, Lewko J, Krajewska K, et al. How to live with a chronic rheumatic disease - nurse's role in patient education.[Jak żyć z przewlekłą chorobą reumatyczną - rola pielęgniarki w edukacji pacjentów]. Probl Pielęg. 2008; 16(1, 2): 199-204.

7. Sierakowska M, Doroszkiewicz H, Nyklewicz W, Mojsa W.Recommendations of the European League Against Rheumatism - implications for nursing care. [Rekomendacje Europejskiej Ligi Przeciwreumatycznej - implikacje dla opieki pielęgniarskiej]. Probl Pielęg. 2010; 18(2): 232-238.

8. Hill J, Tennant A, Adebajo A. Further development of an educational needs assessment tool (ENAT) for patients with rheumatoid arthritis. Arthritis Rheum. 2004; 50: 274-275.

9. Ndosi M, Tennant A, Bergsten U, et al. Cross-cultural validation of the Educational Needs Assessment Tool in RA in 7 European countries. BMC Musculoskelet Disord. 2011; 12(1): 110.
10. Ndosi M, Bremander A, Hamnes B, et al. Validation of the educational needs assessment tool as a generic instrument for rheumatic diseases in seven European countries. AnnRheum Dis. 2014; 73: 2122-2129. doi: 10.1136/annrheumdis-2013-203461.

11. Beaton DE, Bombardier C, Guillemin F, Ferraz MB. Guidelines for the process of cross-cultural adaptation of self-report measures. Spine 2000; 25(24): 3186-3191.

12. Sierakowska M, Sierakowski S, Sierakowska J, et al. Developing the Polish Educational Needs Assessment Tool (Pol-ENAT) in rheumatoid arthritis and systemic sclerosis: a cross-cultural validation study using Rasch analysis. Qual Life Res. 2014; 24(3): 721-733.

13. Smolen JS, Landewé R, Breedveld FC, et al.EULAR recommendations for the management of rheumatoid arthritis with synthetic and biological disease-modifying drugs. Ann Rheum Dis. 2010; 69: 964-975.

14. Grønning K, Rannestad T, Skomsvoll JF, et al. Long - term effects of a nurse-led group and individual patient education programme for patients with chronic inflammatory polyarthritis - a randomized controlled trial. J Clin Nurs. 2013. doi:10.1111/jocn.12353.

15. van Eijk-Hustings Y, van Tubergen A, Boström C, et al. EULAR recommendations for the role of the nurse in the management of chronic inflammatory arthritis. Ann Rheum Dis. 2012; 71:13-19. doi: 10.1336/annrheumdis-2011-200185.

16. Rupiński R, Filipowicz-Sosnowska A. Disease activity and disability in patients with rheumatoid arthritis. [Aktywność choroby a niepełnosprawność u chorych na reumatoidalne zapalenie stawów]. Reumatologia 2005; 43(3): 129-137.

17. Ambriz Murillo Y, Menor Almagro R, Campos-González ID, Cardiel $\mathrm{MH}$. Health related quality of life in rheumatoid arthritis, osteoarthritis, diabetes mellitus, end stage renal disease and geriatric subjects. Experience from a General Hospital in Mexico. Reumatol Clin. 2015 Mar-Apr; 11(2): 68-72. doi: 10.1016/j.reuma.2014.03.006.

18. Wysocka-Skurska I, Sierakowska M, Sierakowski S. Evaluation of the quality of life of patients with rheumatoid arthritis depending on the used therapy. [Ocena jakości życia pacjentów z reumatoidalnym zapaleniem stawów w zależności od stosowanej terapii farmakologicznej]. Rheumatologia 2012; 50(1): 16-23.

19. van Eijk-Hustings Y, Ammerlaan J, Voorneveld-Nieuwenhuis H, et al. Patient' needs and expectations with regard to rheumatology nursing care: results of multicenter focus group interviews. Ann Rheum Dis. 2013; 72 (6): 831-835. doi: 10.1136/annrheumdis-2012-202810.

20. Drăgoi RG, Ndosi $M$, Sadlonova $M$, et al. Patient education, disease activity and physical function: can we be more targeted? A cross sectional study among people with rheumatoid arthritis, psoriatic arthritis and hand osteoarthritis. Arthritis Res Ther. 2013, 15:R156, http://arthritis-research.com/content/15/5/R156.

21. Neame R, Hammond A, Dieghton C. Need for information and for involvement in decision making among patients with rheumatoid arthritis: a questionnaire survey. Arthritis Rheum. 2005; 53: 249-255.

22. Adab P, Rankin EC, Witney AG, et al. Use of a corporate needs assessment to define the information requirements of an arthritis resource centre in Birmingham: comparison of patients' and professionals views. Rheumatol. (Oxford) 2004; 43: 1513-1518.

23. Meesters J, Vliet Vlieland T, Hill J, Ndosi M. Measuring educational needs among patients with rheumatoid arthritis using the Dutch version of the Educational Needs Assessment Tool (DENAT). Clin Rheumatol. 2009; 28: 1073-1077.

24. Isik A, Koca S, Ozturk A, Mermi O. Anxiety and depression in patients with rheumatoid arthritis. Clin Rheumatol. 2007; 26: 872-878.

25. Pytel A, Wrzosek Z. Estimation of patient knowledge on rheumatoid arthritis in the range of their own disease - preliminary study. Adv Clin Exp Med. 2012; 21(3): 343-351.

26. Arvidsson S, Bergman S, Arvidsson B, et al. Effects of a self-care promoting problem-based learning programme in people with rheumatic diseases: a randomized controlled study. J Adv Nurs. 2013; 69,7: 1500-1514. doi: 10.1111/jan 12008 . 